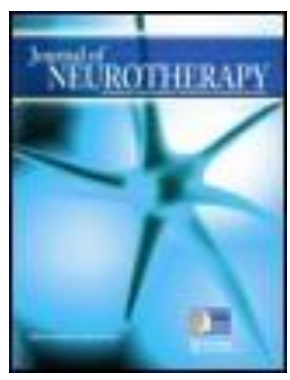

Journal of Neurotherapy: Investigations in Neuromodulation, Neurofeedback and Applied Neuroscience

\title{
The Effects of Brief, Eyes-Open Alpha Brain Wave Training with Audio and Video Relaxation Induction on the EEG of 77 Army Reservists
}

\author{
John Putman MS \\ Published online: 17 Oct 2008.
}

To cite this article: John Putman MS (2000) The Effects of Brief, Eyes-Open Alpha Brain Wave Training with Audio and Video Relaxation Induction on the EEG of 77 Army Reservists, Journal of Neurotherapy: Investigations in Neuromodulation, Neurofeedback and Applied Neuroscience, 4:1, 17-28, DOI: $\underline{10.1300 / J 184 v 04 n 01 \_03}$

To link to this article: http://dx.doi.org/10.1300/J184v04n01_03

\section{PLEASE SCROLL DOWN FOR ARTICLE}

(c) International Society for Neurofeedback and Research (ISNR), all rights reserved. This article (the "Article") may be accessed online from ISNR at no charge. The Article may be viewed online, stored in electronic or physical form, or archived for research, teaching, and private study purposes. The Article may be archived in public libraries or university libraries at the direction of said public library or university library. Any other reproduction of the Article for redistribution, sale, resale, loan, sublicensing, systematic supply, or other distribution, including both physical and electronic reproduction for such purposes, is expressly forbidden. Preparing or reproducing derivative works of this article is expressly forbidden. ISNR makes no representation or warranty as to the accuracy or completeness of any content in the Article. From 1995 to 2013 the Journal of Neurotherapy was the official publication of ISNR (www. Isnr.org); on April 27, 2016 ISNR acquired the journal from Taylor \& Francis Group, LLC. In 2014, ISNR established its official open-access journal NeuroRegulation (ISSN: 2373-0587; www.neuroregulation.org).

THIS OPEN-ACCESS CONTENT MADE POSSIBLE BY THESE GENEROUS SPONSORS

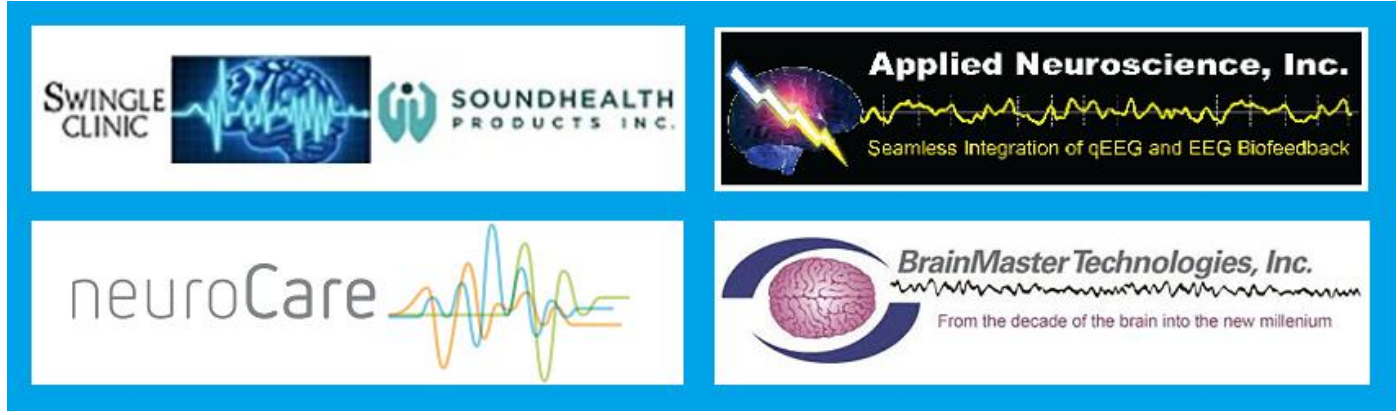




\title{
The Effects of Brief, Eyes-Open Alpha Brain Wave Training with Audio and Video Relaxation Induction on the EEG of 77 Army Reservists
}

\author{
John Putman, MS
}

\begin{abstract}
Background: Recently, psychologist Barry Sterman of the UCLA School of Medicine became involved in measuring the brain wave activity of pilots engaged in a variety of tasks for the purpose of identifying the brain wave correlates of peak performance under different load conditions. Sterman found that during a manageable periodic challenge the brain waves exhibited, in parietal areas, a consistent cycling between resting state alpha (when in the attentive readiness state) and an alpha desynchronized, elevated low beta state when engaged in the response mode. As the tasks came closer together, hence allowing for no alpha respite, there was a deterioration in performance accompanied by an increase in theta activity. In this paper, the effects of brief, eyes-open alpha brain wave enhancement training will be examined for the general purpose of suggesting possible methods for increasing functional integrity and cortical flexibility through increased alpha brain wave production.
\end{abstract}

Methods: The subjects were 77 U.S. Army reservists. The EEG

John Putman, MS, is a licensed psychotherapist and BCIA certified biofeedback therapist specializing in EEG biofeedback. He is currently in private practice in Los Angeles. His education includes an MA in Clinical Psychology, a BA in Mathematics and an MS in Electromedical Science.

Address correspondence to: John Putman, 1710 N. Fuller Avenue, \#507, Hollywood, CA 90046 (E-mail: JPutman905@aol.com).

The author would like to thank EEG Spectrum Inc. for providing the instrumentation used in this project and Siegfried Othmer, $\mathrm{PhD}$ for review of an earlier manuscript. He is also grateful to David Kaiser, $\mathrm{PhD}$ for the graphical representations of the data and his useful feedback. Final thanks go to Dr. and Mrs. Fred Detwiler for their courage in attempting to do the near impossible. 
biofeedback system used was the BioIntegrator manufactured by the Bio-Research Institute. Alpha enhancement training was employed with electrode placement at Pz.

Results: It was found that eyes-open alpha enhancement training resulted in substantial increases in activity in the feedback band (alpha) with smaller increases in low beta and decreases in theta. This is quite a different result than one would expect from general "relaxation" training that is usually accomplished with eyes closed and yields substantial increases in both alpha and theta.

Conclusion: When engaged in this training, even for brief periods, the EEG moves in a direction quite different to that of Sterman's burnout profile. It would be of interest to demonstrate rigorously that the training could, if administered preventatively, diminish poor performance in persons performing tasks that demand prolonged periods of external focus under high load conditions.

KEYWORDS. Eyes-open alpha, PRS, Post Reinforcement Synchrony, cortical flexibility, alpha immersion

\section{INTRODUCTION}

There is a rather long history of measuring brain waves and associating them with various states of consciousness from sleep to hyperarousal. Many states of consciousness defy categorization from an EEG standpoint, but the basic states and their associated EEG frequencies are as follows: High Beta $(18-30 \mathrm{~Hz})$ associated with intense focus and hyper arousal, Low Beta $(12-18 \mathrm{~Hz})$ associated with external attentiveness and moderate arousal, Alpha $(8-12 \mathrm{~Hz})$ associated with relaxation and passive attention, Theta $(4-8 \mathrm{~Hz})$ associated with deep relaxation and inward focus and Delta $(.5-3 \mathrm{~Hz})$ associated with sleep. Brain waves are reflected micro changes in electrical potential usually measured at the scalp's surface. They represent synchronous firings of neurons located in specific areas of the cortex. Although the EEG contains no useful information about the specific "content" of cognitive processes or of thoughts in general, it does manifest changes in the state of physiological arousal, of attentive focus, and even of mood. Over the past thirty years or so, researchers have demonstrated that teaching a person to deliberately alter their EEG, through such 
techniques as operant conditioning via EEG biofeedback, can be very effective in treating problems involving disregulation in the dimensions of arousal, attention and affect. Well established benefits have been seen, for example, in the treatment of epilepsy (Sterman and MacDonald, 1978) and attention deficit disorder (Lubar and Shouse, 1976; Lubar and Lubar, 1984).

Recently, psychologist Barry Sterman of the Sepulveda Veterans Administration and the UCLA School of Medicine, became involved in a NASA sponsored project measuring the brain wave activity of high performance jet pilots engaged in a variety of tasks for the purpose of identifying the brain wave correlates of peak performance under various operational conditions. A number of tests were performed to measure response capability under a wide range of conditions (from boring vigilance tasks to those of increasing complexity and potential task overload). Pilots were given a relatively easy task or situation that would eventually progress in difficulty beyond human ability to maintain an appropriate response. Sterman (1994) discovered that pilots who were able to maintain their alpha activity during flight simulation demonstrated more accuracy in task performance than those whose alpha activity decreased. Additionally, he found that during a manageable and periodic challenge the brain waves (in parietal sites) exhibited a consistent cycling between resting state alpha (the attentive readiness state) and an alpha-desynchronized, elevated low beta state when engaged in responding. They would then move back into the alpha state following withdrawal of the challenging stimuli. This resting state alpha proved absolutely necessary for the maintenance of optimum performance. This alpha activity is referred to as Post Reinforcement Synchrony (PRS), in that it represented a momentary respite, or pleasure response, following successful completion of a challenge. (Such a challenge has historically been presented in an operant conditioning paradigm, in which the PRS was first identified-hence the reference to reinforcement.) As tasks became increasingly complex, the pilots required longer and longer periods of alpha synchrony before being able to respond to a challenging situation. Further, as the tasks came closer together in time, hence allowing for no alpha respite between challenges, performance fell off. After dealing with these demanding situations for an extended period of time, the pilots' brain wave activity began to exhibit higher and higher amounts of theta-associated, in this case, with nonfunctionality and 
burnout. Thus, the ability to punctuate stressful periods of high arousal with an alpha reward appears to be necessary in order to maintain adequate performance and avoid burn out. Although the theta state, with its internal focus, can be replenishing for the hyper aroused brain, it can become a dangerous sanctuary in situations that demand continuous external attention and focus. Sterman's work on peak performance implies a basic training protocol which would: (a) Expand and strengthen flexibility by training the capacity to alter the thalamic arousal and attention set point to fit the circumstances; (b) Aid the smooth transitioning between states and the flexibility to maintain the appropriate state when under challenge; and (c) Specifically reinforce the PRS cycle, especially the ability to enter the "readiness" of resting state alpha. (Campbell, 1995).

It is likely that those persons with adequate PRS are more resistant to trauma inducing stress since their brains already possess the flexibility to move from one level of arousal to another. This would lead to greater ease in processing traumatic experiences. Those with the burnout profile (high amplitude theta, low alpha \& high beta) seem unable to navigate the full spectrum of arousal needed to handle and recover from stressful situations. It is likely that it is this flexibility in the brain that determines how effectively an individual processes and recovers from stressful events.

In this paper, the effects of brief alpha brain wave training with audio and video relaxation induction (collectively referred to as 'alpha immersion') on the EEG of several adults will be examined. During 1996-1997, data was collected as part of a series of demonstrations involving an anti-stress and neurolab mobile clinic under the direction of Lt. Colonel Fred S. Detwiler, MD, a psychiatrist in the U.S. Army Reserve. The unit is intended for use in emergency situations for persons suffering from emotional trauma and was deployed once in an actual emergency following the 1994 Northridge earthquake. Several demonstrations of this unit have been given around the country, primarily at hospitals and military medical conferences. During this time, a great deal of raw EEG data was collected on numerous military personnel while they were engaged in brief, eyes open alpha brain wave biofeedback training augmented with relaxation induction. Data was recorded on over 100 people (only 77 records were useable) for the purpose of examining the effectiveness of brief alpha immersion training on facilitating increases in alpha activity. Changes in alpha 
amplitudes over the session were used as a measure of a change in state. Increases in the amplitude of specific brain wave frequencies associated with relaxation (alpha, 8-12 Hz) and decreases in those associated with hyper arousal, acute anxiety and fatigue (beta, 13-42 $\mathrm{Hz}$ ), were expected.

The subjects received visual feedback on the alpha activity only; hence this will be the primary area of interest. The percent change over the course of the training (which was about 12 minutes in duration for each person) was calculated and examined in such measures as alpha amplitude, beta amplitude, theta amplitude and the alpha/ beta and theta/beta ratios. The differences in the scatter of male and female over age with respect to these measures were examined. Additionally, hours of sleep per night were correlated with changes over these bandwidths. The primary goal was to analyze amplitude changes in the feedback band (alpha) and compare them to changes in the nonfeedback bands, (theta and beta). The clinical significance of the amplitude measure is well established. According to Robert Thatcher, the relevance of EEG amplitude is related to the fact that the output of a population of EEG generators is a function of the number of generators, the synchrony of the generators and the geometry of the generators (Thatcher and John, 1977; Nunez, 1981).

\section{METHODS}

Subjects. The subjects were 77 attendees at three consecutive combat stress and medical conferences over the period of a year and a half. The participants were primarily U.S. Army reservists between the ages of 25 and 67 (male and female). This was not a randomized sample, but rather a sample of convenience.

Procedure. The computerized EEG biofeedback system used was the BioIntegrator, manufactured by the Bio-Research Institute. Included is a battery-operated encoder (the ProComp from Thought Technology) with optical cable for data stream transmission. The system utilizes 8 input channels, two of which are dedicated to the EEG measurement. The EEG program uses Fourier transforms for continuous signal processing that provide a fairly refined spectral display of the activity (frequency bins were $.78 \mathrm{~Hz}$ wide). For feedback purposes the frequency bin of greatest value was considered representative of its associated band. For example, the greatest amplitude change in the 
alpha band took place at around $10 \mathrm{~Hz}$. Data was recorded in 12 one-minute epochs. The numeric data (representing the average per epoch of all frequency bins for a particular band) were examined in alpha, theta and beta bands over the twelve-minute period. A referenced placement was used with the signal electrode located at $\mathrm{Pz}$ according to the international 10-20 system. Ground and reference electrodes were placed on each ear. The volunteer was instructed to sit in a comfortable recliner and view a stress reduction video containing natural scenery accompanied by a relaxation-inducing narrative. A second video screen was placed immediately above the first, which provided a visual display (in the form of a multicolored mandala) of the individual's alpha brain wave activity $(8-12 \mathrm{~Hz})$. The feedback was direct in that the intensity of the mandala varied directly with alpha amplitude. The mandala would fill in visually in direct proportion to the alpha amplitude for a specific scale (in this case 0 to 30 microvolts). For example, the screen would be black at 0 microvolts and completely filled at 30 . The audio feedback was disabled as this would have interfered with the narrative. Each participant was instructed to keep their eyes open and view the video tape while glancing at the feedback screen approximately every ten seconds. The two screens were kept close together and in the line of sight in order to facilitate easy alternate viewing of both screens.

\section{RESULTS}

Statistical analysis of the data on pre-post changes in EEG amplitude revealed that there were no significant dependences on age and on gender. Hence, for present purposes, the data was collapsed on those variables. The distribution of change scores is shown in terms of histograms in the three bands evaluated: alpha, beta and theta (Figures $1 \mathrm{a}, \mathrm{b}$ and $\mathrm{c}$, respectively).

The mean change and standard error of the mean are shown in Figure 2 for the three bands. The changes in the alpha and beta bands are seen to be significant. The change in the feedback band (alpha) is the largest change observed. It appears that increasing alpha amplitudes results in substantial changes in those amplitudes and in the adjacent bands as well. It seems that percent of alpha change and percent of beta change are highly and directly correlated with most of the beta increase taking place between 12 and $20 \mathrm{~Hz}$ (Figure 3). The 
FIGURE 1a. Effect of EEG Biofeedback Training

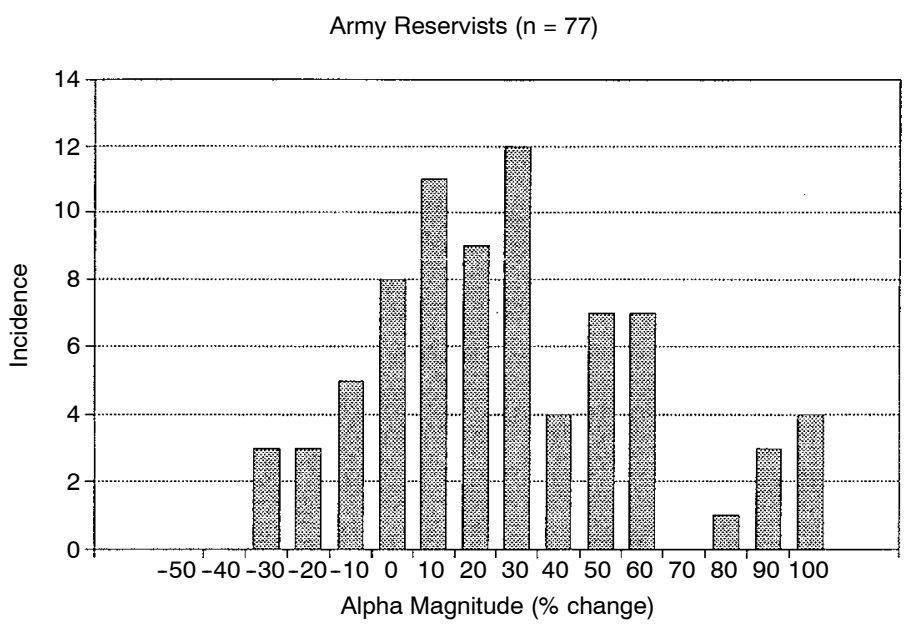

FIGURE 1b. Effect of EEG Biofeedback Training

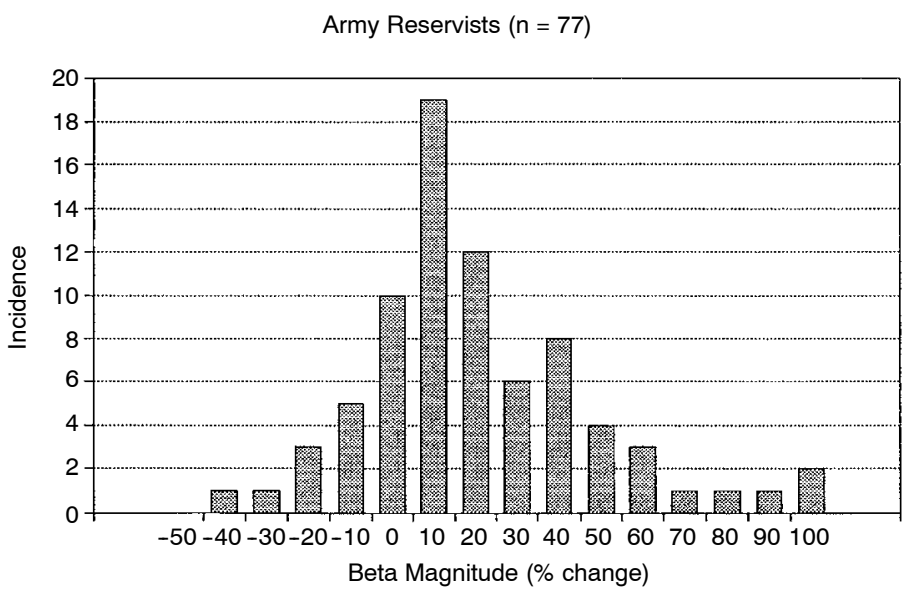

default setting for beta activity on this instrument was $12-42 \mathrm{~Hz}$. While amplitudes increased in the alpha and low beta band, theta amplitudes decreased. This can be understood in terms of a particular state of arousal that was being promoted by the training. It is expected that alpha amplitudes should increase in the eyes-closed condition. 
FIGURE 1c. Effect of EEG Biofeedback Training

Army Reservists $(\mathrm{n}=77$ )

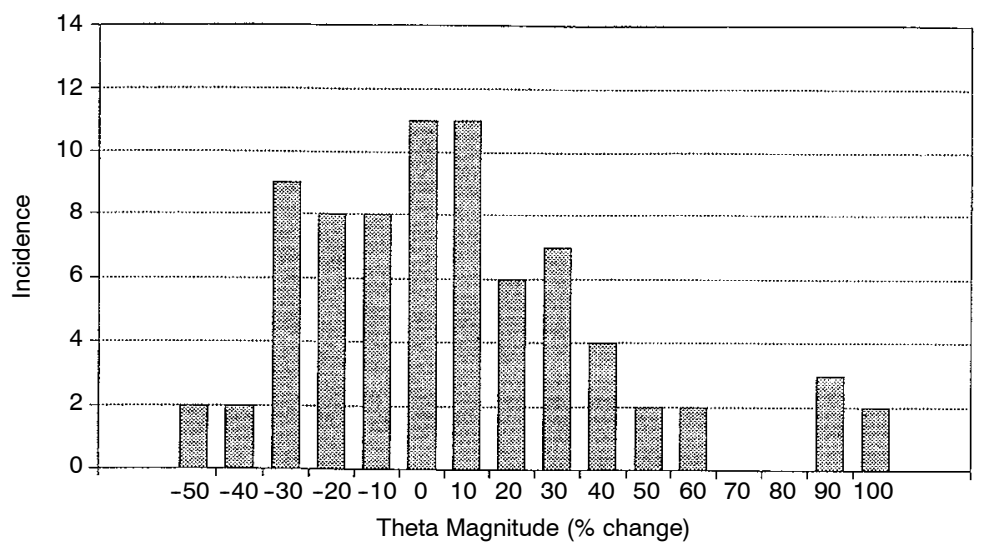

FIGURE 2. Effect of EEG Biofeedback Training

Army Reservists $(\mathrm{n}=77$ )

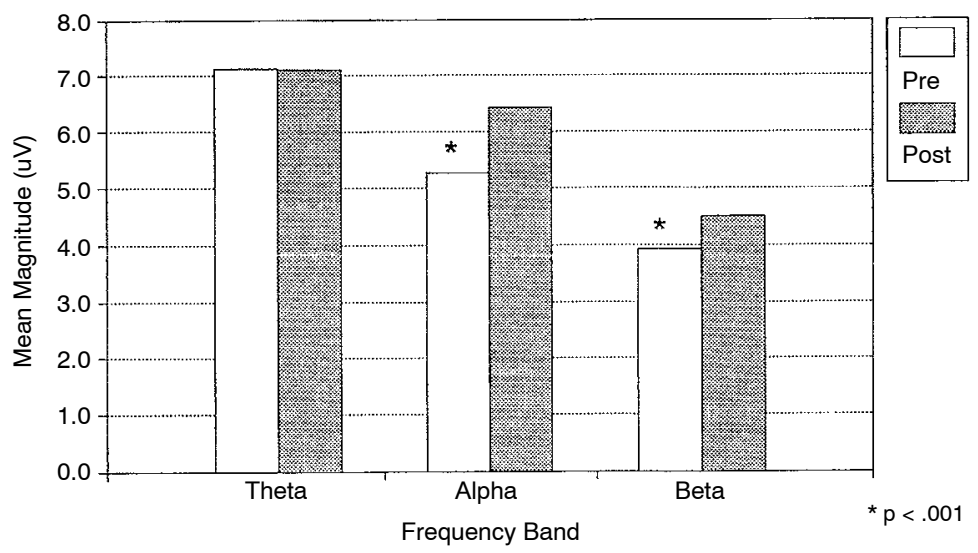

However, EEG reinforcement training and exposure to relaxation imagery required that the eyes remain open. Hence, the training apparently did not simply promote a lowering of arousal, leading perhaps to drowsiness. Higher theta amplitudes, contrary to observation, would have accompanied such a state. Since we were training with the eyes 
FIGURE 3. Effect of EEG Biofeedback Training

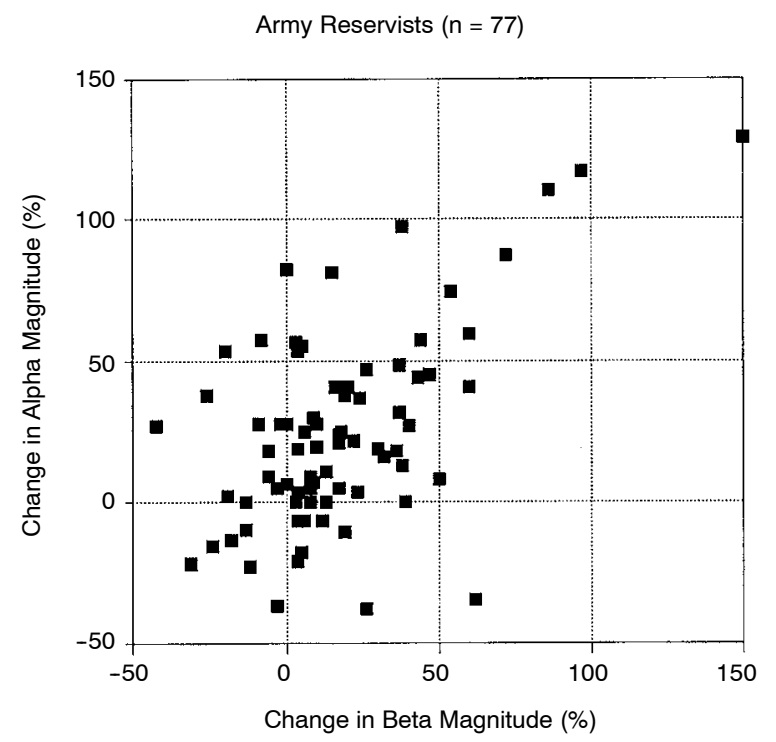

open, which is essentially training against a challenge (i.e., visual stimulation), it is likely that additional mechanisms of cortical control and arousal were being engaged. Stimulation of the visual cortex is sufficient to cause desynchronization of low frequency activity resulting in lower theta amplitudes. Therefore, it is tempting to conclude that the selective reinforcement of alpha amplitude under eyes-open conditions promotes a very specific state of arousal as opposed to a general lowering of arousal level, as might be implied by the term "relaxation."

Upon questioning, nearly all of the participants reported feelings of well being following the training. Some even reported experiencing pleasant memories from childhood. All reported an increase in their energy level. Prior sleep history, surprisingly, did not seem to play a major role in the performance of this particular sample population. Eleven subjects had a decrease in their mean alpha amplitudes. It is known that persons who are predisposed toward external vigilance often experience "letting go" as threatening. The idea of relinquishing control may induce anxiety leading to a "paradoxical" decrease in alpha activity. Whether the data presented in these figures represent a change in state could not be determined in the present context, but it would be of interest for future investigation. 


\section{DISCUSSION}

The data displayed in Figure 3 suggests a possible one-to-one correspondence between percent of alpha change and percent of beta change (if one were to draw a line connecting the origin with the centroid of the primary cluster it would yield a vector with a slope of approximately one). It would be of interest to see if the addition of more data points resulted in an increase in the density of this cluster with the limit of the arithmetic mean falling on or close to this vector. While alpha and low beta activity tended to increase together, theta activity tended to decrease. The average percentage change in theta was -10 while the mean amplitude showed no change (Figure 2). It appears that even though we were giving feedback in a discrete band (8-12 Hz in this case), the brain seems to have its own attenuation agenda with regard to its EEG output in that feeding back alpha activity with eyes open results in an increase in alpha amplitude with a shallow roll off into the higher frequencies and a sharp drop off at the low end into theta. Additionally, the dominant alpha frequency remained relatively unchanged at $10 \mathrm{~Hz}$ over the twelve-minute interval and, as such, was not an effective measure of a change in state.

These results may provide further evidence for the argument that targeting specific frequency bands for reinforcement doesn't necessarily mean that changes in the EEG will be confined to those discrete areas. This is particularly true when enhancing midrange activity at or near the sensory motor cortex (Pz in this case). The most effective reward bands are those that facilitate fundamental changes through the activation of subcortical regulatory mechanisms that virtually guarantee non-linear manifestations in the EEG. When doing neurofeedback we are giving the brain a task: increase the alpha amplitude at location " A", for example. The brain can accomplish this task in a number of ways. In the frontal areas it may tend to rely on local recruitment to do the job while closer to the sensory motor strip it will engage subcortical loops resulting in shifts in parameters not directly being trained such as phase and coherence.

The reported effects of brief eyes-open alpha enhancement training seem quite similar to SMR training which is not too surprising since the SMR band (which, at $12-15 \mathrm{~Hz}$, is adjacent to the alpha band) increased along with the alpha activity. Enhancing a specific part of the midrange frequency $(8-18 \mathrm{~Hz})$ may result in a kind of cortical liberation that manifests as an increase in activity along the entire 
band. The fact that the percentage change in alpha and beta were so directly correlated may suggest activation of the same agent of change (e.g., proportionate numbers of neurons being activated at the respective frequencies by some common mechanism). Such a mechanism would likely yield percent changes of similar magnitude with less similarity between amplitude changes.

Brief eyes-open alpha reinforcement, in an immersed environment, has been shown to yield EEG changes consistent with achievement of a more relaxed state even with brief exposure. Unfortunately, the design does not allow one to discern the relative contributions of the environment (videotape, relaxation induction audio track, background music, protective environmental chamber) and of the explicit alpha amplitude reinforcement to the overall changes in the EEG amplitudes. Additionally, although the subjects were instructed to observe the feedback six times per minute, the actual number of observations seemed to vary between approximately five and twenty per one-minute epoch. In retrospect, it may have been useful to compare total time attending to the feedback with the degree of change observed in the alpha, beta and theta amplitudes. This may have helped to differentiate between the relative contributions of the various elements of the alpha immersion chamber. Unfortunately, such data were not recorded. On the basis of these initial findings, however, it may be conjectured that more extensive brain wave training in such an environment may be effective in combating stress related problems, such as deteriorating performance, in persons involved in work that demands sustained attention. This could occur by increasing the capacity for experiencing post reinforcement synchrony that has been shown to be conducive to the maintenance of functional integrity under challenge. When engaged in this training, even for brief periods, the EEG appears to move in the direction opposite to that of Sterman's burnout profile (i.e., increased theta and beta with diminished midrange alpha activity). It would be of interest to demonstrate rigorously that the training couldif administered in a prophylactic manner-diminish fatigue, anxiety and poor performance in persons involved in high stress occupations that demand prolonged periods of external focus of attention under high load conditions. These may include air traffic controllers, pilots, police, emergency care providers, military personnel, etc.

The concept of cortical flexibility raises other important questions. Sterman views the thalamically mediated PRS cycle as critical in 
determining levels of arousal and attentional capacity. The thalamus, in effect, serves as a "weighting" function that prioritizes various incoming stimuli for action by the cortex. There are also return projections from the cortex to the thalamus that allow the cortex to play a role in regulating itself by impacting thalamically driven cortical field potentials. Is it possible, given the extensive neurocircuitry, that a fatigued cortex (i.e., one deprived of PRS over an extended period of time) impacts the oscillatory set points in the thalamus such that cortical theta activity becomes driven by and sustained by the new sub cortical homeostasis? Such a relationship cannot be established by current research but may be of interest in future investigations. Such an investigation could have important implications regarding the causal role of violence and social tension in attention deficit and learning disorders.

\section{REFERENCES}

Campbell, D. (1995). Toward a philosophy of optimum performance. Mega Brain Report. 3 (1), 52-58.

EEG Spectrum Training Course Manual. (1997). Encino, CA: EEG Spectrum.

Kilch, L.G., McComas, A.J., Osselton, J.W. \& Upton, A.R.M. (1981). Clinical Electroencephalography, Fourth Edition. London: Butterworths

Lubar, J.F., \& Shouse, M.N. (1976). EEG and behavioral changes in a hyperactive child concurrent training of the sensory motor rhythm (SMR). A preliminary report. Biofeedback and Self-Regulation, (1), 293-306.

Lubar, J.O., \& Lubar, J.F. (1984). Electroencephalographic biofeedback of SMR and beta for the treatment of attention deficit disorders in the clinical setting. Biofeedback and Self-Regulation, 9, 1-23.

Nunez, P. (1981). Electrical Fields of the Brain. Cambridge, MA: Oxford University Press.

Sterman, M.B., \& MacDonald, L.R. (1978). Effects of central cortical EEG feedback training on seizure incidence in poorly controlled epileptics. Epilepsia, 19, 207-222.

Sterman, M.B., Mann, C.A., Kaiser, D.A., \& Suyenbu, B.Y., (1994). Multiband topographic EEG analysis of a simulated visuomotor aviation task. International Journal of Psychophysiology, 16, 49-56.

Thatcher, R.W. \& John, E.R. (1977). Functional Neuroscience, Vol. 1: Foundations of Cognitive Processes. E.R. John \& R.W. Thatcher (Eds.) NJ: L. Erlbaum Associates.

RECEIVED: 04/15/99

REVISED: 08/15/99

ACCEPTED: $10 / 29 / 99$ 